\title{
Pharmacokinetic Profiles of Ticagrelor Orodispersible Tablets in Healthy Western and Japanese Subjects
}

\author{
Renli Teng ${ }^{1}$ - Maria Hammarberg ${ }^{2}$ - Glenn F. Carlson ${ }^{1} \cdot$ Sara Bokelund-Singh $^{3}$ • \\ Terese Ruderfelt ${ }^{3} \cdot$ Eva Blychert $^{2}$
}

Published online: 30 August 2017

(c) The Author(s) 2017. This article is an open access publication

\begin{abstract}
Background and Objectives Ticagrelor is an antiplatelet agent for patients with acute coronary syndrome or a history of myocardial infarction. Two studies compared pharmacokinetic profiles of orodispersible (OD) ticagrelor tablets versus immediate-release (IR) tablets in Western and Japanese subjects.

Methods Both studies were open-label, randomized, crossover, single-center trials. Thirty-six healthy subjects (94\% white, $6 \%$ other race; Western study NCT02400333) and 42 Japanese healthy subjects (Japanese study NCT02436577) received a single 90-mg ticagrelor dose as an OD tablet [with/without water, and via a nasogastric tube (Western study only)], and an IR tablet; washout between treatments was $\geq 7$ days. Assessments included ticagrelor and AR-C124910XX (active metabolite) plasma concentrations for pharmacokinetic analyses, and safety evaluations.

Results In the Western study, the $90 \%$ confidence intervals (CIs) of the geometric mean ratios (GMRs) for ticagrelor and AR-C124910XX maximum plasma concentration $\left(C_{\max }\right)$ and area under the plasma concentration-time curve
\end{abstract}

Electronic supplementary material The online version of this article (doi:10.1007/s40261-017-0554-8) contains supplementary material, which is available to authorized users.

Renli Teng

renli.teng@astrazeneca.com

1 AstraZeneca LP, One MedImmune Way, Gaithersburg, MD, USA

2 AstraZeneca Pharmaceutical Technology and Development, Gothenburg, Sweden

3 AstraZeneca Global Medicines Development, Gothenburg, Sweden
(AUC) were within the acceptance interval (80\%-125\%) for OD tablets (with/without water, via a nasogastric tube) versus the IR tablet; except for an $\sim 15 \%$ lowering of ticagrelor $C_{\max }(90 \%$ CI: $76.77 \%-93.78 \%)$ for the OD tablet taken with water. In the Japanese study, 90\% CIs of the GMRs for AUC and $C_{\max }$ of both ticagrelor and ARC124910XX were all within the acceptance intervals for the OD (with/without water) versus IR tablet. No new safety issues were identified.

Conclusions Ticagrelor administered as an OD tablet to Western (without water, and via a nasogastric tube) and Japanese (with/without water) subjects was bioequivalent to the IR tablet.

\section{Key Points}

Ticagrelor, a $\mathrm{P}_{2} \mathrm{Y}_{12}$ receptor inhibitor, is approved as an antiplatelet agent in patients with acute coronary syndrome (90 mg twice daily with low-dose aspirin for the first year) or a history of myocardial infarction (60 mg twice daily with low-dose aspirin, 1 year after an acute coronary syndrome event).

Some patients requiring ticagrelor may have difficulty to swallow whole immediate-release (IR) tablets; thus, a ticagrelor orodispersible (OD) tablet has been developed.

Bioequivalence was demonstrated between OD tablets and IR tablets in healthy Western subjects (without water, and via a nasogastric tube) and in healthy Japanese subjects (with/without water). 


\section{Introduction}

Ticagrelor is an orally administered, direct-acting, reversibly binding $\mathrm{P} 2 \mathrm{Y}_{12}$ receptor antagonist. The mechanism of action of ticagrelor involves the inhibition of ADP-induced platelet aggregation [1-3], and the inhibition of the equilibrative nucleoside transporter 1 , thereby reducing adenosine cellular uptake $[3,4]$.

In the Platelet Inhibition and Patient Outcomes (PLATO) trial, ticagrelor (180 mg loading dose, $90 \mathrm{mg}$ twice daily thereafter) plus aspirin significantly reduced the rate of the primary composite endpoint [myocardial infarction (MI)/stroke/death from vascular causes] in patients with acute coronary syndrome (ACS) versus clopidogrel (300-600 mg loading dose, $75 \mathrm{mg}$ once daily thereafter) plus aspirin, with no difference in the overall rate of major bleeding [5]. In the prospective Prevention of Cardiovascular Events in Patients with Prior Heart Attack Using Ticagrelor Compared to Placebo on a Background of Aspirin-Thrombolysis in Myocardial Infarction 54 (PEGASUS-TIMI 54) study, patients with a prior MI (1-3 years previously) received either ticagrelor (60 or $90 \mathrm{mg}$ twice daily) or placebo and low-dose aspirin [6]. Findings from the PEGASUS-TIMI 54 study showed that long-term therapy (median follow-up 33 months) with ticagrelor significantly reduced the risk of $\mathrm{MI} /$ stroke/death from vascular causes versus placebo [6]. Rates of TIMI major bleeding were higher with ticagrelor versus placebo, although similar rates of intracranial hemorrhage or fatal bleeding were seen across the groups [6]. Based on results of the PLATO and PEGASUS-TIMI 54 studies, ticagrelor is approved to reduce the rate of cardiovascular death, MI, and stroke in patients with ACS (180 mg loading dose, then $90 \mathrm{mg}$ twice daily together with low-dose aspirin for the first year after the ACS event) or a history of MI (60 mg twice daily together with low-dose aspirin from 1 year after the ACS event) [7]. Ticagrelor is recommended in several major US and EU guidelines as an antiplatelet therapy for patients with ST-elevation ACS $[8,9]$ or non-ST elevation ACS [10, 11].

Oral ticagrelor is rapidly absorbed and has a linear pharmacokinetic (PK) profile $[12,13]$, and the major, active metabolite is AR-C124910XX [3, 14, 15]. Plasma concentrations of AR-C124910XX are approximately $30 \%-40 \%$ of ticagrelor plasma concentrations [3]. Both ticagrelor and AR-C124910XX are reversible $\mathrm{P}_{2} \mathrm{Y}_{12}$ receptor antagonists, and AR-C124910XX has a similar potency to ticagrelor in inhibiting the $\mathrm{P}_{2} \mathrm{Y}_{12}$ receptor [3]. The main pharmacodynamic effect, platelet inhibition, is rapid, temporary, and related to plasma concentrations of both ticagrelor and AR-C124910XX [3, 12, 13, 16, 17]. In Asian subjects, mean bioavailability of ticagrelor and AR-
C124910XX is $\sim 40 \%$ higher versus Caucasian subjects [18-21]. However, in the Asia $(n=1056)$ and China $(n=383)$ subgroups of the PLATO study, the efficacy and safety of ticagrelor versus clopidogrel were generally consistent with the overall study population [5]. In the Phase Three International study of Ticagrelor and Clinical Outcomes in Asian ACS patients (PHILO) study in Japanese, Korean and Taiwanese patients with ACS, treatment with standard doses of ticagrelor resulted in higher event rates of the primary safety and efficacy endpoints versus clopidogrel, the differences between the groups were not statistically significant [22].

Certain patients who cannot take standard tablets require alternative formulations [23], e.g. unconscious, intubated patients, those with certain co-morbidities, critically ill patients (e.g. $15 \%$ of survivors of critical illness, $51 \%$ of cardiac surgery patients on mechanical ventilation $>48 \mathrm{~h}$ [24] have swallowing difficulties), and the elderly (e.g. $15 \%-20 \%$ of the elderly population in the USA [25]). Furthermore, dysphagia, a common problem in adults ( $\sim 4 \%$ incidence in the USA [26]), may result in drug administration errors [27]. Such patients who are unable to take tablets encompass those with cardiovascular disease [28-31] who may need antiplatelet therapy.

It is well recognized that drug formulations impact pharmacokinetic parameters [32]. For ticagrelor, bioequivalence was demonstrated for crushed versus whole tablets in healthy subjects [33]. The ticagrelor prescribing information notes that crushed ticagrelor tablets can be used for patients with swallowing difficulties [7]. However, crushing tablets is often inconvenient and requires access to water. An orodispersible (OD) tablet would overcome these issues, and would be suitable for patients with swallowing difficulties and who are unable to swallow whole tablets. OD tablets differ from traditional tablets as they are designed to dissolve or disintegrate on the tongue rather than being swallowed whole [34, 35]. Several different processes, such as freeze-drying, cotton-candy process, spray drying, mass extrusion and compaction, are available for preparing OD tablets that rapidly disintegrate in the mouth [35]. In addition, excipients to aid the feel of the OD tablet in the mouth also contribute to disintegration [35]. Other advantages of OD tablets include convenience of dosing and improved patient compliance [34]. Given that OD tablets offer several advantages for patients with swallowing difficulties, and have the convenience of a tablet that can be taken without water, such formulations have an important role in oral drug delivery $[34,35]$.

Given the clinical need for a ticagrelor formulation suitable for patients unable or unwilling to swallow tablets, an OD 90-mg tablet formulation of ticagrelor has been developed (Fig. 1). Compared with the immediate-release 


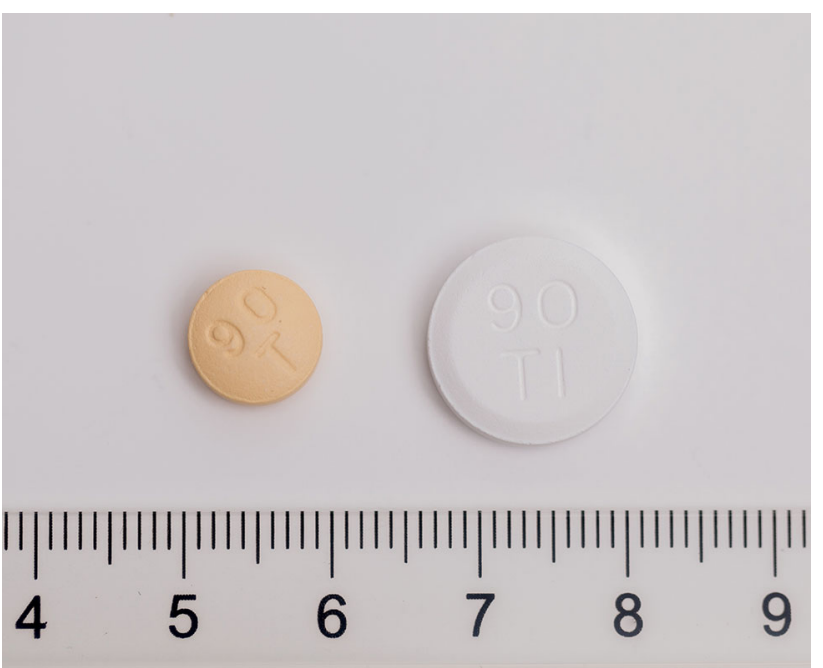

Fig. 1 Ticagrelor OD tablet (white) and IR tablet (yellow). IR immediate release, $O D$ orodispersible

(IR) tablet, the ticagrelor OD tablet contains excipients to aid disintegration on the tongue and to give a smooth feeling in the mouth, which facilitates swallowing of the dispersed tablet. Also, the OD tablet is non-coated and has a more porous structure that facilitates water ingress to further enhance rapid disintegration.

Two studies in healthy subjects were conducted with ticagrelor OD tablets. One study recruited mainly white, healthy subjects (referred to as the Western study herein) and the other study was in Japanese, healthy subjects. Both studies assessed the pharmacokinetic profiles of ticagrelor OD tablets versus the currently approved formulation of IR tablets.

\section{Methods}

\subsection{Study Designs and Treatments}

The Western study was an open-label, randomized, fourperiod, four-treatment, crossover, single-center, singledose study (NCT02400333) (Fig. 2a). The Japanese study was an open-label, randomized, three-period, three-treatment, crossover, single-center, single-dose study (NCT02436577) (Fig 2b).

In both studies, subjects were randomized to a treatment sequence on day 1 of the first treatment period; the Western study had four treatment sequences (Fig. 2a) and the Japanese study had six treatment sequences (Fig. 2b). Randomization codes were assigned strictly sequentially as the subjects became eligible. For each treatment period, ticagrelor was administered on day 1 after an overnight fast. The three treatments that were used in both studies were: a single 90-mg ticagrelor OD tablet administered with water (the OD tablet was placed on the tongue to disintegrate then swallowed with noncarbonated water at room temperature); a single 90-mg ticagrelor OD tablet administered without water (the OD tablet was disintegrated on the tongue then swallowed with saliva); and a single 90-mg ticagrelor IR tablet administered with water. In the Western study only, a single 90-mg ticagrelor OD tablet suspended in water was administered via a nasogastric tube with water. The total volume of water used to administer ticagrelor was $200 \mathrm{~mL}$ (Western study) or $150 \mathrm{~mL}$ (Japanese study). The washout period between treatments in each study was at least 7 days.

\subsection{Study Populations}

For both studies, healthy men and women (non-pregnant, not lactating, post-menopausal or surgically sterile) were eligible for inclusion. Japanese subjects were defined as those who had both parents and four grandparents who were Japanese. For the Western subjects, the age and body mass index (BMI) ranges were 18-55 years and $18.5-29.9 \mathrm{~kg} / \mathrm{m}^{2}$, respectively. For the Japanese subjects, the age and BMI ranges were 20-45 years and $18.0-27.0 \mathrm{~kg} / \mathrm{m}^{2}$, respectively. The minimum and maximum body weight permitted were $\geq 50$ to $\leq 100 \mathrm{~kg}$ (Western subjects) and $\geq 45$ to $\leq 85 \mathrm{~kg}$ (Japanese subjects).

In both studies, subjects were excluded for the following key reasons: a history or presence of any condition known to interfere with drug absorption, distribution, metabolism or excretion; a known or suspected history of alcohol and/ or drug abuse; plasma or blood donation or blood loss $>500 \mathrm{~mL}$ during the 3 months prior to screening; use of any drug or presence/history of any condition known to increase the propensity for bleeding for 2 weeks before randomization; platelet count $<150 \times 10^{9} / \mathrm{L}$; current smokers; previous smoking or nicotine patch use in the previous 3 months; and use of enzyme inducers within the previous 3 weeks.

\subsection{Study Assessments}

\subsubsection{Pharmacokinetics}

In both studies, $2 \mathrm{~mL}$ blood samples were collected at 0 (pre-dose), and 0.5, 1, 2, 3, 4, 6, 8, 10, 12, 16, 24, 36, and $48 \mathrm{~h}$ after each treatment dose. Harvested plasma samples were stored at $\leq-20{ }^{\circ} \mathrm{C}$ until analysis.

Plasma concentrations of ticagrelor and ARC124910XX were analyzed using a fully validated, liquid chromatography-tandem mass spectrometry method [36]. The range of the calibration curves for ticagrelor and ARC124910XX were $5-5000 \mathrm{ng} / \mathrm{mL}$ and $2.5-2500 \mathrm{ng} / \mathrm{mL}$, respectively. The mean intra-batch precision and accuracy 
Fig. 2 Flow chart showing the treatment sequences and periods for the a Western study, and b Japanese study. Treatment $\mathrm{A}=\mathrm{a}$ single $90-\mathrm{mg}$ ticagrelor OD tablet administered with water; Treatment $\mathrm{B}=\mathrm{a}$ single 90-mg ticagrelor OD tablet administered without water; Treatment C (Western study) = a single $90-\mathrm{mg}$ ticagrelor OD tablet suspended in water administered via a nasogastric tube with water; Treatment C (Japanese study)/ Treatment D (Western study) = a single $90-\mathrm{mg}$ ticagrelor IR tablet administered with water. $I R$ immediate release, $O D$ orodispersible a

Sequence

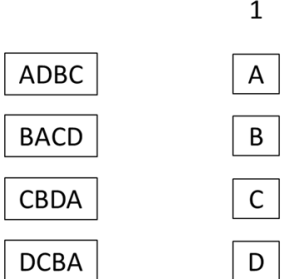

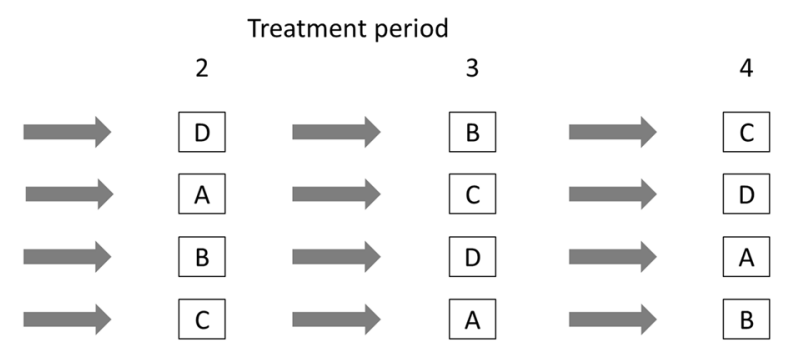

b

Sequence

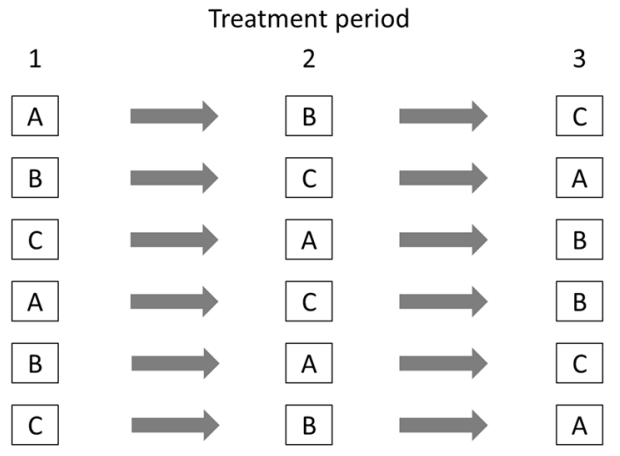

of this method is $4.0 \%-8.4 \%$ and $91.9 \%-109.0 \%$, respectively, for ticagrelor, and 5.2\%-16.9\% and 86.8\%-109.2\%, respectively, for AR-C124910XX [36].

\subsubsection{Safety and Tolerability}

Adverse events (AEs) were recorded continuously throughout both studies (for further details, see Supplementary methods). At screening and follow-up, all subjects had a physical examination, a vital signs check (blood pressure and pulse), a 12-lead electrocardiogram, and an evaluation of clinical laboratory parameters [hematology, clinical chemistry, and urinalysis (dipstick)]. Vital signs were also assessed during each treatment period at pre-dose $(0 \mathrm{~h})$ and post-dose at 2,4 , and $24 \mathrm{~h}$.

\subsection{Data Analyses}

\subsubsection{Sample Sizes}

Based on the results of a previous ticagrelor study (drugdrug interaction with diltiazem [37]), the within-subject coefficient of variations for maximum plasma concentration $\left(C_{\max }\right)$, the area under the plasma-concentration time curve (AUC) from zero to infinity $\left(\mathrm{AUC}_{0-\infty}\right)$ for ticagrelor and AR-C124910XX were assumed to be $\leq 24 \%$ for both compounds. To achieve a statistical power of $90 \%$ that a twosided $90 \%$ confidence interval (CI) for the ratio of $C_{\max }$ between two different ticagrelor treatments would be contained within the $0.80-1.25$ limit, 28 (using a four-sequence
Williams design for four periods and four treatments) and 30 (using a six-sequence Williams design for three periods and three treatments) evaluable subjects would be required in the Western and Japanese studies, respectively. To allow for withdrawals, two subjects were added to each sequence. Thus, up to 36 and 42 subjects were randomized in the Western and Japanese studies, respectively.

\subsubsection{Pharmacokinetic Analyses}

Ticagrelor and AR-C124910XX pharmacokinetic parameters were calculated using standard non-compartmental methods (WinNonlin ${ }^{\circledR}$ Professional, Pharsight Corporation, Mountain View, CA, USA). Key pharmacokinetic parameters were: $\mathrm{AUC}_{0-\infty} ; \mathrm{AUC}_{0-t} ; C_{\max }$; time to $C_{\max }\left(t_{\max }\right)$; terminal half-life $\left(t_{1 / 2}\right)$; and metabolite:parent ratios of $C_{\max }$, $\mathrm{AUC}_{0-\infty}$, and $\mathrm{AUC}_{0-\mathrm{t}}$. Pharmacokinetic data were summarized by treatment group using descriptive statistics.

Within each study, statistical comparisons between the OD tablet, for each administration route, and the IR tablet (reference) were performed on the difference of logtransformed $C_{\max }, \mathrm{AUC}_{0-\infty}$, and $\mathrm{AUC}_{0-\mathrm{t}}$ of both ticagrelor and AR-C124910XX. An analysis of variance model including fixed effects for treatment, sequence, period and subject within sequence was used to estimate the treatment differences with $90 \%$ CIs. The estimates were back transformed to obtain the geometric mean ratios (GMRs) for each pair of treatments. The criteria for acceptance was for the $90 \%$ CIs of the GMRs to be within $80 \%-125 \%$ [38-40]. 


\section{Results}

\subsection{Subjects}

\subsubsection{Western Study}

Thirty-six healthy subjects were randomized. Four subjects were withdrawn due to protocol deviations and two subjects withdrew consent. Thus, 30 subjects completed all four treatment periods. Overall, of the randomized subjects, $32(89 \%)$ were male, and $34(94 \%)$ were white and $2(6 \%)$ were of another race. The mean \pm standard deviation (SD) age and BMI were $42 \pm 12$ years and $24.6 \pm 2.4 \mathrm{~kg} / \mathrm{m}^{2}$, respectively.

\subsubsection{Japanese Study}

Forty-two Japanese healthy subjects were randomized and one was withdrawn due to a protocol deviation. Thus, 41 subjects completed all three treatment periods. All randomized subjects were male, and the mean \pm SD age and BMI were $31 \pm 7$ years and $21.8 \pm 2.0 \mathrm{~kg} / \mathrm{m}^{2}$, respectively.

\subsection{Pharmacokinetics}

\subsubsection{Western Study}

Plasma concentration-time curves for ticagrelor (Fig. 3a) and AR-C124910XX (Fig. 3b) following administration of an OD tablet with or without water, or administered via a nasogastric tube were generally similar to those following administration of an IR tablet.

With the OD tablet (regardless of administration method) and the IR tablet, ticagrelor was rapidly absorbed with a median $t_{\max }$ of $\sim 2 \mathrm{~h}$, and the mean $t_{1 / 2}$ was $\sim 8 \mathrm{~h}$. AR-C124910XX was rapidly formed with a median $t_{\max }$ of 2-3 $\mathrm{h}$, and the mean $t_{1 / 2}$ was $\sim 9 \mathrm{~h}$ (Table 1 ).

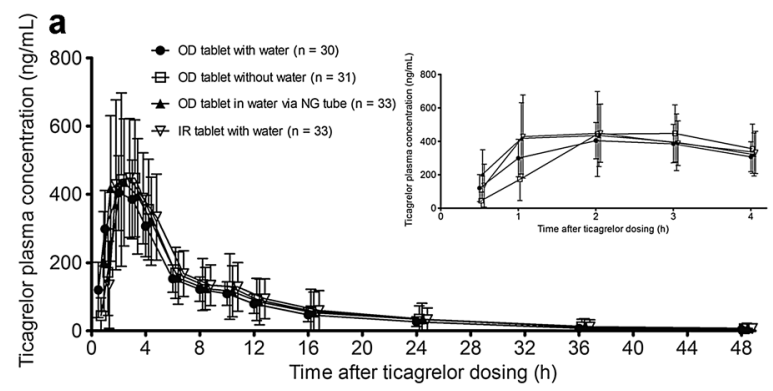

Fig. 3 Arithmetic mean ( \pm SD) plasma concentrations of a ticagrelor and b AR-C12491XX over time following administration of a 90-mg dose of ticagrelor as an OD tablet (with and without water; suspended
Comparison of the OD tablet (regardless of administration) with the IR tablet showed that the $90 \%$ CIs of the GMRs for ticagrelor $\mathrm{AUC}_{0-\infty}$ and $\mathrm{AUC}_{0-\mathrm{t}}$ were within $80 \%-125 \%$. The $90 \%$ CIs of the GMRs for ticagrelor $C_{\max }$ were also within this acceptance range for the OD tablet taken without water, or administered via a nasogastric tube versus the IR tablet. However, ticagrelor $C_{\max }$ for the OD tablet taken with water was $\sim 15 \% \quad(90 \%$ CI 76.77-93.78\%) lower than with the IR tablet (Table 1). For AR-C124910XX, the $90 \%$ CIs of the GMRs for $\mathrm{AUC}_{0-\infty}$, $\mathrm{AUC}_{0-\mathrm{t}}$ and $C_{\max }$ were all within $80 \%-125 \%$ for the OD tablet (regardless of administration) versus the IR tablet (Table 1). The low-to-moderate between-subject variability was similar across treatments for ticagrelor and ARC124910XX. The coefficient of variation of the GMR was $\sim 25-34 \%$ for $C_{\max }$, and $\sim 29 \%-44 \%$ for AUC for both compounds.

\subsubsection{Japanese Study}

Plasma concentration-time curves for ticagrelor (Fig. 4a) and AR-C124910XX (Fig. 4b) following administration of an OD tablet with or without water were generally similar to those following administration of an IR tablet.

With the OD tablet with and without water and the IR tablet, ticagrelor was rapidly absorbed with a median $t_{\max }$ of $2-3 \mathrm{~h}$, and the mean $t_{1 / 2}$ was $\sim 8 \mathrm{~h}$. AR-C124910XX was rapidly formed with a median $t_{\max }$ of $\sim 3 \mathrm{~h}$, and the mean $t_{1 / 2}$ was $\sim 9 \mathrm{~h}$ (Table 2).

For both ticagrelor and AR-C124910XX, the 90\% CIs of the GMRs for $\mathrm{AUC}_{0-\infty}, \mathrm{AUC}_{0-\mathrm{t}}$ and $C_{\max }$ were all within $80-125 \%$ for the OD tablet, with and without water, versus the IR tablet (Table 2).

\subsection{Safety and Tolerability}

In both studies, no deaths, serious AEs or AEs leading to study discontinuation occurred. There were no clinically

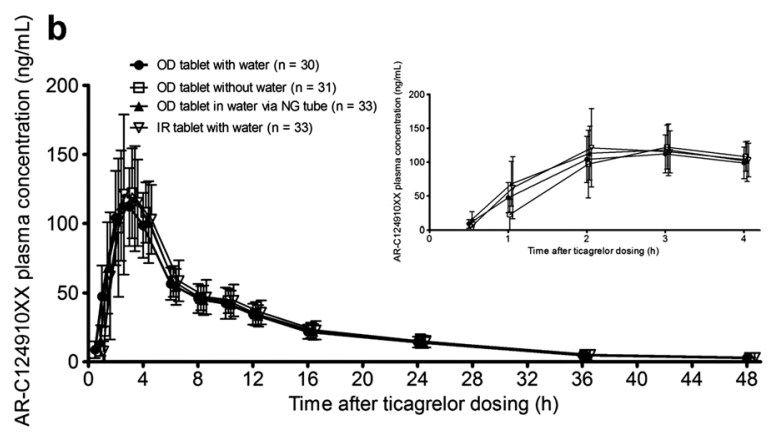

in water administered via a nasogastric tube), or an IR tablet to Western healthy subjects. $I R$ immediate release, $N G$ nasogastric, $O D$ orodispersible, $S D$ standard deviation 


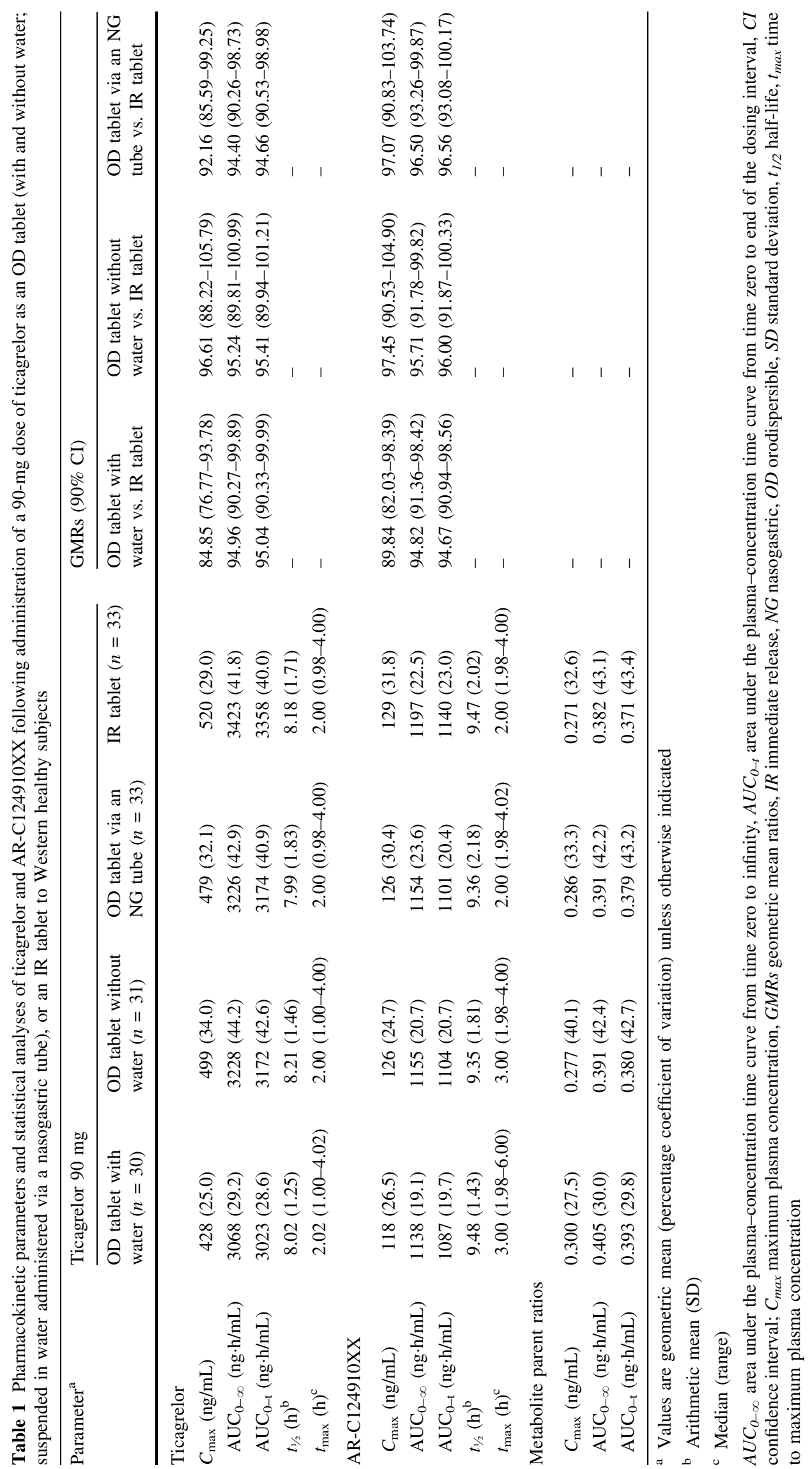




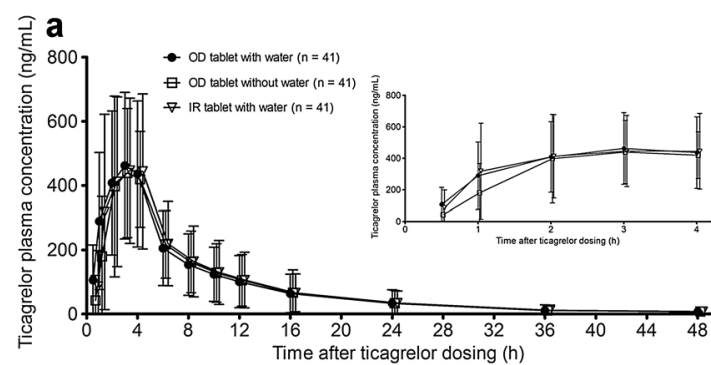

Fig. 4 Arithmetic mean ( $\pm \mathrm{SD}$ ) plasma concentrations of a ticagrelor b AR-C12491XX over time following administration of a 90-mg dose of ticagrelor as an OD tablet (with and without water), or an IR tablet

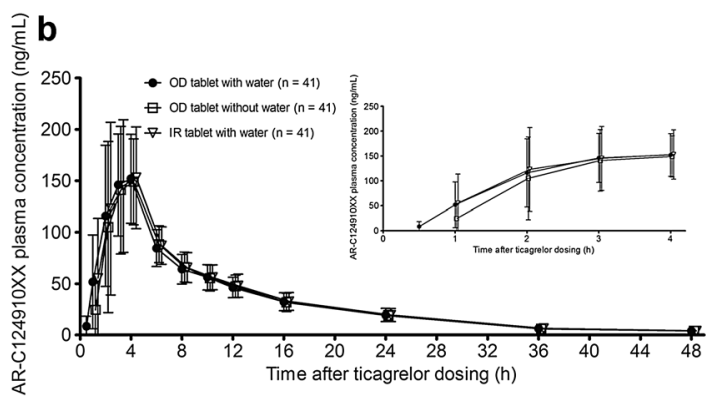

to Japanese healthy subjects. $I R$ immediate release, $O D$ orodispersible, $S D$ standard deviation

Table 2 Pharmacokinetic parameters and statistical analyses of ticagrelor and AR-C124910XX following administration of a 90-mg dose of ticagrelor as an OD tablet (with and without water), or an IR tablet to Japanese healthy subjects

\begin{tabular}{|c|c|c|c|c|c|}
\hline \multirow[t]{2}{*}{ Parameter $^{\mathrm{a}}$} & \multicolumn{3}{|l|}{ Ticagrelor $90 \mathrm{mg}$} & \multicolumn{2}{|l|}{ GMRs $(90 \%$ CI) } \\
\hline & $\begin{array}{l}\text { OD tablet with } \\
\text { water }(n=41)\end{array}$ & $\begin{array}{l}\text { OD tablet without } \\
\text { water }(n=41)\end{array}$ & $\begin{array}{l}\text { IR tablet } \\
(n=41)\end{array}$ & $\begin{array}{l}\text { OD tablet with water } \\
\text { vs. IR tablet }\end{array}$ & $\begin{array}{l}\text { OD tablet without } \\
\text { water vs. IR tablet }\end{array}$ \\
\hline \multicolumn{6}{|l|}{ Ticagrelor } \\
\hline$C_{\max }(\mathrm{ng} / \mathrm{mL})$ & $529(38.4)$ & $534(29.8)$ & $569(37.0)$ & $93.16(85.80-101.15)$ & 93.67 (87.88-99.84) \\
\hline $\mathrm{AUC}_{0-\infty}(\mathrm{ng} \cdot \mathrm{h} / \mathrm{mL})$ & $3520(45.1)$ & $3485(42.8)$ & $3606(46.3)$ & $97.75(94.40-101.21)$ & $96.50(93.31-99.80)$ \\
\hline $\mathrm{AUC}_{0-\mathrm{t}}(\mathrm{ng} \cdot \mathrm{h} / \mathrm{mL})$ & $3462(43.8)$ & $3423(41.4)$ & $3546(45.0)$ & $97.76(94.46-101.18)$ & 96.38 (93.24-99.63) \\
\hline$t_{1 / 2}(\mathrm{~h})^{\mathrm{b}}$ & $7.74(1.19)$ & $7.94(1.19)$ & $7.86(1.09)$ & - & - \\
\hline$t_{\max }(\mathrm{h})^{\mathrm{c}}$ & $3.00(1.00-4.02)$ & $3.00(1.02-5.97)$ & $2.00(1.00-6.00)$ & - & - \\
\hline \multicolumn{6}{|l|}{ AR-C124910XX } \\
\hline$C_{\max }(\mathrm{ng} / \mathrm{mL})$ & $165(31.4)$ & $158(36.5)$ & $170(35.5)$ & $96.79(88.27-106.14)$ & $92.32(85.04-100.23)$ \\
\hline $\mathrm{AUC}_{0-\infty}(\mathrm{ng} \cdot \mathrm{h} / \mathrm{mL})$ & $1547(23.3)$ & $1503(24.0)$ & $1573(22.3)$ & $98.46(94.85-102.20)$ & 95.47 (91.99-99.08) \\
\hline $\mathrm{AUC}_{0-\mathrm{t}}(\mathrm{ng} \cdot \mathrm{h} / \mathrm{mL})$ & $1488(23.5)$ & $1441(24.5)$ & 1513 (22.7) & $98.43(94.75-102.25)$ & 95.15 (91.59-98.85) \\
\hline$t_{1 / 2}(\mathrm{~h})^{\mathrm{b}}$ & $9.13(1.91)$ & $9.12(1.81)$ & $9.05(1.68)$ & - & - \\
\hline$t_{\max }(\mathrm{h})^{\mathrm{c}}$ & $3.07(2.00-6.00)$ & $3.00(2.00-6.02)$ & $3.00(1.00-8.00)$ & - & - \\
\hline \multicolumn{6}{|l|}{ Metabolite parent ratios } \\
\hline$C_{\max }(\mathrm{ng} / \mathrm{mL})$ & $0.340(41.4)$ & $0.322(44.8)$ & $0.327(43.2)$ & - & - \\
\hline $\mathrm{AUC}_{0-\infty}(\mathrm{ng} \cdot \mathrm{h} / \mathrm{mL})$ & $0.480(41.8)$ & $0.471(42.9)$ & $0.476(43.6)$ & - & - \\
\hline $\mathrm{AUC}_{0-\mathrm{t}}(\mathrm{ng} \cdot \mathrm{h} / \mathrm{mL})$ & $0.469(42.3)$ & $0.460(43.1)$ & $0.466(44.0)$ & - & - \\
\hline
\end{tabular}

${ }^{a}$ Values are geometric mean (percentage coefficient of variation) unless otherwise indicated

b Arithmetic mean (SD)

c Median (range)

$A U C_{0-\infty}$ area under the plasma-concentration time curve from time zero to infinity, $A U C_{0-t}$ area under the plasma-concentration time curve from time zero to end of the dosing interval, $C I$ confidence interval; $C_{\max }$ maximum plasma concentration, GMRs geometric mean ratios, $I R$ immediate release, $O D$ orodispersible, $S D$ standard deviation, $t_{1 / 2}$ half-life, $t_{\max }$ time to maximum plasma concentration

relevant changes in laboratory parameters, physical examinations, vital signs or ECGs during either study.

In total, 18 mild AEs, regardless of causality, were reported in 9 Western subjects. The numbers (\%) of subjects with at least one AE were 3 (9.7\%), 5 (15.6\%), 3 $(8.8 \%)$ and $2(6.1 \%)$ with the ticagrelor OD tablet with water, without water, or via a nasogastric tube, or the IR tablet, respectively. Overall, the AEs (regardless of causality) occurring in more than one subject were dizziness $(n=2)$ and thrombophlebitis $(n=2)$. Seven mild, treatment-related AEs, identified by the investigator, were reported in five Western subjects. The numbers (\%) of subjects with at least one treatment-related $\mathrm{AE}$ were 1 (3.2\%), $3(9.4 \%), 1(2.9 \%)$ and $2(6.1 \%)$ with the ticagrelor OD tablet with water, without water, or via a nasogastric tube, or the IR tablet, respectively. All treatment-related AEs only occurred in one subject (i.e. dizziness, headache, chest pain, fatigue, dyspnea, hematoma, and rash). 
In Japanese subjects, a total of 15 AEs (all mild or moderate in severity), regardless of causality, were reported in 7 subjects. The numbers (\%) of subjects with at least one AE were $3(7.3 \%), 4(9.5 \%)$, and $3(7.3 \%)$ with the ticagrelor OD tablet with water, or without water, or the IR tablet, respectively. Overall, the AEs (regardless of causality) occurring in more than one subject were contusion $(n=3)$, dizziness $(n=2)$, headache $(n=2)$, and rhinorrhea $(n=2)$. Six treatment-related AEs, identified by the investigator, were reported in four Japanese subjects. The numbers $(\%)$ of subjects with at least one treatmentrelated AE were $2(4.9 \%), 3(7.1 \%)$, and 1 (2.4\%) with the ticagrelor OD tablet with water, without water, or the IR tablet, respectively. The treatment-related AEs occurring in one subject only were dizziness, rhinorrhea, oropharyngeal pain, feeling hot, and pyrexia. Treatment-related headache was reported in two Japanese subjects.

\section{Discussion}

The main objective of the present studies was to compare the bioavailability of ticagrelor following administration of either an OD tablet or an IR tablet. The pharmacokinetic findings for ticagrelor and AR-C124910XX demonstrated that most comparisons were within the acceptance interval for OD versus IR tablets, except for ticagrelor $C_{\max }$ following administration of the OD tablet with water in Western subjects. The lower boundary of the $90 \%$ CI for this parameter was outside the acceptance range of $80 \%-$ $125 \%$ [38-40]. The reason for the lower $C_{\max }$ of ticagrelor following administration of the OD tablet with water in Western subjects is unknown. In response to a European Medicines Agency (EMA) request, key pharmacokinetic data from the Western and Japanese studies were pooled. Data pooling fulfils the EMA guideline recommendation to consider all the evidence as a whole if multiple studies have been conducted with a given formulation, and some results show bioequivalence, whereas other results do not [39]. As both the Western and Japanese studies had a crossover design, and, therefore, each subject was its own internal control, pooling data from both studies is considered appropriate. Additionally, due to the crossover design, any between-subject differences in age and body weight/ BMI, for example, would have no impact on $C_{\max }$ and AUC for tablet comparisons. Based on the pooled data (Supplementary Methods), the ticagrelor OD tablet given with or without water was bioequivalent to the IR tablet as the $90 \%$ CIs of the GMRs for AUC and $C_{\max }$ of both ticagrelor and AR-C124910XX were within the acceptance range of $80 \%-125 \%$ (Supplementary Table S1). Thus, overall, the results from both the present studies, and the pooled data analyses, indicate that the OD ticagrelor tablet, with or without water, could be used in place of an IR tablet.

The validity of the present studies is confirmed since the current findings are in keeping with previously reported data. The pharmacokinetics and safety following a single dose of ticagrelor have been extensively studied, including studies using the same ticagrelor IR tablet employed in the present studies [41]. In the present Western study, the ticagrelor and AR-C124910XX pharmacokinetic parameters are generally comparable to previous studies in Caucasian subjects with a single 90-mg ticagrelor dose $[12,13]$. Furthermore, the pharmacokinetic parameters in the current Japanese study are also generally similar to previous studies in Asian subjects, including Chinese [18] and Japanese [19] healthy subjects and in Japanese, Asian [20], and Chinese patients with coronary artery disease [21]. With regards to safety and tolerability, a single, 90-mg dose of ticagrelor was well tolerated in both studies. Moreover, the AE profile of ticagrelor in the present studies was consistent with previous studies in Caucasian subjects [5, 12, 13, 17], and Asian subjects [18-21]. No new safety concerns with ticagrelor were identified with the OD tablet versus the IR tablet.

The present studies have some limitations. Only a single, 90-mg ticagrelor dose was evaluated, whereas, clinically, the ticagrelor dosing regimen is a 180-mg loading dose and $90 \mathrm{mg}$ twice daily thereafter [7]. Furthermore, the current results in healthy subjects may not reflect those in populations of patients with ACS. In addition, the pharmacodynamic effects (i.e. platelet inhibition) were not evaluated in these studies.

The current pharmacokinetic results with the ticagrelor OD tablet, demonstrating bioequivalence with the IR tablet, are clinically important. OD tablets potentially address the medical needs of patients who are unable to take standard tablets [23, 34, 35]. Such patients include those who are unconscious, sedated, critically ill [24], elderly [25], or have dysphagia [26]. Moreover, all these scenarios are likely to be applicable to patients with cardiovascular disease [28-31] who may require antiplatelet therapy. Based on the current results, the ticagrelor OD tablet is considered a suitable alternative formulation to the 90-mg ticagrelor IR tablet for patients who are unable to swallow whole tablets, for whom there is a preference for an OD formulation, or for those who require nasogastric administration. Easy dispersal of an OD tablet in water is simpler than crushing tablets for nasogastric tube administration. Other advantages of a ticagrelor OD tablet include convenience of dosing and use in situations when no water is available [34, 35].

Alternative methods of administering ticagrelor have also been studied, i.e. crushing or chewing the standard tablets. An ex vivo study demonstrated the feasibility of 
administering crushed ticagrelor tablets either orally or via a nasogastric tube [42]. Subsequently, a healthy subject study showed that crushed ticagrelor tablets were bioequivalent (i.e. pharmacokinetic parameters) to whole tablets [33]. Interestingly, higher ticagrelor and ARC124910XX plasma levels occurred in the first hour post-dosing and $t_{\max }$ values were shorter for crushed versus whole tablets in healthy volunteers [33] and in patients with ST-elevation MI (STEMI) [43]. However, compared with the IR tablet, the ticagrelor OD tablet did not result in higher exposure to ticagrelor or ARC124910XX at early time points post-dosing or a shorter $t_{\max }$ in either of the present studies. Three studies evaluated the effects of chewing ticagrelor tablets, the Chewing Versus Swallowing Ticagrelor to Accelerate Platelet Inhibition in ACS (CHEERS) study in patients with non-STEMI, the CHEERS-STEMI (similar study to CHEERS, but in patients with STEMI) and the Inhibition of Platelet Aggregation After Administration of Three Different Ticagrelor Formulations (IPAAD-Tica) study in patients with stable angina. All these studies showed that chewing two $90 \mathrm{mg}$ ticagrelor tablets (180 $\mathrm{mg}$ loading dose) resulted in a faster onset of platelet inhibition versus a standard oral loading dose with whole tablets [44-46]. However, none of these studies reported ticagrelor or AR-C124910XX pharmacokinetic parameters. A faster onset of platelet inhibition in patients with STEMI has also been reported for crushed versus whole ticagrelor tablets [43, 47]. However, as platelet inhibition was not evaluated in the current studies, it is inappropriate to speculate on the possible pharmacodynamic effects of the ticagrelor OD tablet.

\section{Conclusions}

Following a single 90-mg dose of ticagrelor as an OD tablet to Western (with or without water, or via a nasogastric tube) or Japanese (with or without water) healthy subjects, pharmacokinetic parameters of ticagrelor and AR-C124910XX were comparable with those following administration of an IR tablet. The $90 \%$ CIs of the GMRs of $C_{\max }$ and AUC for both compounds were within 80\%-125\%; except for ticagrelor $C_{\max }$ following administration of the OD tablet with water in the Western study, which was $\sim 15 \%$ lower than the $C_{\text {max }}$ with the ticagrelor IR tablet. These results indicate that ticagrelor administered as an OD tablet to Western (without water, and via a nasogastric tube) and Japanese (with/without water) subjects was bioequivalent to the IR tablet. The safety profile of OD and IR ticagrelor tablets in both studies was consistent with previous studies.
Acknowledgements The authors thank Mats Någård for his assistance with protocol preparation. Medical writing assistance (outline, all drafts, assembling tables and figures, collating author comments, grammatical editing and referencing) was provided by Jackie Phillipson, PhD (Zoetic Science, an Ashfield company, part of UDG Healthcare plc, Macclesfield, UK); this assistance was funded by AstraZeneca.

Author contributions RT, GC and SB-S contributed to the conception and design (protocol development and/or design advice), acquisition of data, data analyses, and data interpretation. MH, TR and EB contributed to the conception and design (protocol development and/or design advice), and data interpretation. All authors critically reviewed the drafts of the manuscript and provided approval of the final version submitted.

\section{Compliance with Ethical Standards}

Funding These studies were funded by AstraZeneca.

Conflict of interest RT, TR and EB are employees of AstraZeneca. $\mathrm{MH}$ and GC are employees of AstraZeneca and hold AstraZeneca stock/stock options. SB-S is a full time, paid consultant (Statistician) for AstraZeneca.

Ethics Both study protocols and the protocol amendment (Western study only) were reviewed and approved by independent Ethics committees (Western study: State Office of Health and Social Affairs, Ethics Committee of the Land Berlin, Berlin, Germany; Japanese study: NRES Committee East of England-Cambridgeshire and Hertfordshire, East of England-Cambridgeshire and Hertfordshire Research Ethics Committee Centre, Jarrow, Tyne and Wear, UK). Each study was conducted in accordance with the Declaration of Helsinki and the International Conference on Harmonization/Good Clinical Practice Guidelines and followed applicable regulatory requirements and AstraZeneca's policy on bioethics.

Informed consent All subjects in both studies provided informed, written consent prior to any study-specific procedures.

Open Access This article is distributed under the terms of the Creative Commons Attribution-NonCommercial 4.0 International License (http://creativecommons.org/licenses/by-nc/4.0/), which permits any noncommercial use, distribution, and reproduction in any medium, provided you give appropriate credit to the original author(s) and the source, provide a link to the Creative Commons license, and indicate if changes were made.

\section{References}

1. Husted S, van Giezen JJ. Ticagrelor: the first reversibly binding oral P2Y12 receptor antagonist. Cardiovasc Ther. 2009;27:259-74.

2. van Giezen JJ, Nilsson L, Berntsson P, Wissing BM, Giordanetto F, Tomlinson W, Greasley PJ. Ticagrelor binds to human P2Y(12) independently from ADP but antagonizes ADP-induced receptor signaling and PA. J Thromb Haemost. 2009;7:1556-65.

3. Nylander S, Schulz R. Effects of P2Y12 receptor antagonists beyond platelet inhibition-comparison of ticagrelor with thienopyridines. Br J Pharmacol. 2016;173:1163-78.

4. Armstrong D, Summers C, Ewart L, Nylander S, Sidaway JE, van Giezen JJ. Characterization of the adenosine pharmacology of 
ticagrelor reveals therapeutically relevant inhibition of equilibrative nucleoside transporter 1. J Cardiovasc Pharmacol Ther. 2014;19:209-19.

5. Wallentin L, Becker RC, Budaj A, Cannon CP, Emanuelsson H, Held C, et al. Ticagrelor versus clopidogrel in patients with acute coronary syndromes. N Engl J Med. 2009;361:1045-57.

6. Bonaca MP, Bhatt DL, Cohen M, Steg PG, Storey RF, Jensen EC, et al. Long-term use of ticagrelor in patients with prior myocardial infarction. N Engl J Med. 2015;372:1791-800.

7. AstraZeneca LP. Brilinta (ticagrelor) tablets. Approved prescribing information. Revised September 2016. http://www. azpicentral.com/brilinta/brilinta.pdf. Accessed 9 March 2017.

8. O'Gara PT, Kushner FG, Ascheim DD, Casey DE Jr, Chung MK, de Lemos JA, et al. 2013 ACCF/AHA guideline for the management of ST-elevation myocardial infarction: a report of the American College of Cardiology Foundation/American Heart Association Task Force on Practice Guidelines. Circulation. 2013; 127:e362-425.

9. Steg PG, James SK, Atar D, Badano LP, Blömstrom-Lundqvist $\mathrm{C}$, Borger MA, et al. ESC guidelines for the management of acute myocardial infarction in patients presenting with ST-segment elevation. Eur Heart J. 2012;33:2569-619.

10. Amsterdam EA, Wenger NK, Brindis RG, Casey DE Jr, Ganiats TC, Holmes DR Jr, et al. 2014 AHA/ACC guideline for the management of patients with non-ST-elevation acute coronary syndromes: executive summary: a report of the American College of Cardiology/American Heart Association Task Force on Practice Guidelines. Circulation. 2014;130:2354-94.

11. Roffi M, Patrono C, Collet JP, Mueller C, Valgimigli M, Andreotti F, et al. 2015 ESC guidelines for the management of acute coronary syndromes in patients presenting without persistent ST-segment elevation: Task Force for the Management of Acute Coronary Syndromes in Patients Presenting without Persistent ST-Segment Elevation of the European Society of Cardiology (ESC). Eur Heart J. 2016;37:267-315.

12. Teng R, Butler K. Pharmacokinetics, pharmacodynamics, tolerability and safety of single ascending doses of ticagrelor, a reversibly binding oral $\mathrm{P} 2 \mathrm{Y}(12)$ receptor antagonist, in healthy subjects. Eur J Clin Pharmacol. 2010;66:487-96.

13. Butler K, Teng R. Pharmacokinetics, pharmacodynamics, safety and tolerability of multiple ascending doses of ticagrelor in healthy volunteers. Br J Clin Pharmacol. 2010;70:65-77.

14. Teng R, Oliver S, Hayes MA, Butler K. Absorption, distribution, metabolism, and excretion of ticagrelor in healthy subjects. Drug Metab Dispos. 2010;38:1514-21.

15. Zhou D, Andersson TB, Grimm SW. In vitro evaluation of potential drug-drug interactions with ticagrelor: cytochrome P450 reaction phenotyping, inhibition, induction and differential kinetics. Drug Metab Dispos. 2011;39:703-10.

16. Gurbel PA, Bliden KP, Butler K, Tantry US, Gesheff T, Wei C, et al. Randomized double-blind assessment of the ONSET and OFFSET of the antiplatelet effects of ticagrelor versus clopidogrel in patients with stable coronary artery disease: the ONSET/ OFFSET study. Circulation. 2009;120:2577-85.

17. Husted S, Emanuelsson H, Heptinstall S, Sandset PM, Wickens M, Peters G. Pharmacodynamics, pharmacokinetics, and safety of the oral reversible P2Y12 antagonist AZD6140 with aspirin in patients with atherosclerosis: a double-blind comparison to clopidogrel with aspirin. Eur Heart J. 2006;27:1038-47.

18. Li H, Butler K, Yang L, Yang Z, Teng R. Pharmacokinetics and tolerability of single- and multiple-doses of ticagrelor in healthy Chinese volunteers. Clin Drug Investig. 2012;32:87-97.

19. Teng R, Butler K. Comparison of the pharmacokinetics, pharmacodynamics and tolerability of single and multiple doses of ticagrelor in healthy Japanese and Caucasian volunteers. Int $\mathbf{J}$ Clin Pharmacol Ther. 2014;54:478-91.
20. Hiasa Y, Teng R, Emanuelsson H. Pharmacodynamics, pharmacokinetics and safety of ticagrelor in Asian patients with stable coronary artery disease. Cardiovasc Interv Ther. 2014;29:324-33.

21. Li H, Guo J, Carlson GF, Teng R. Pharmacodynamics, pharmacokinetics, and safety of ticagrelor in Chinese patients with stable coronary heart disease. Br J Clin Pharmacol. 2016;82:352-61.

22. Goto S, Huang CH, Park SJ, Emanuelsson H, Kimura T. Ticagrelor vs. clopidogrel in Japanese, Korean and Taiwanese patients with acute coronary syndrome-randomized, double-blind, phase III PHILO study. Circ J. 2015;79:2452-60.

23. Stegemann S, Gosch M, Breitkreutz J. Swallowing dysfunction and dysphagia is an unrecognized challenge for oral drug therapy. Int J Pharm. 2012;430:197-206.

24. Macht M, White SD, Moss M. Swallowing dysfunction after critical illness. Chest. 2014;146:1681-9.

25. Sura L, Madhavan A, Carnaby G, Crary MA. Dysphagia in the elderly: management and nutritional considerations. Clin Interv Aging. 2012;7:287-98.

26. Bhattachryya $\mathrm{N}$. The prevalence of dysphagia among adults in the United States. Otolaryngol Head Neck Surg. 2014;151:765-9.

27. Wright DJ, Kelly J. Medication administration in patients with dysphagia. Nurs Stand. 2012;27:35-40.

28. Jackson CF, Wenger NK. Cardiovascular disease in the elderly. Rev Eps Cardiol. 2011;64:697-712.

29. Rab T, Kern KB, Tamis-Holland JE, et al. Cardiac arrest: a treatment algorithm for emergent invasive cardiac procedures in the resuscitated comatose patient. J Am Coll Cardiol. 2015;66:62-73.

30. Grimm J, Magruder T, Ohkuma R, et al. A novel risk score to predict dysphagia after cardiac surgery procedures. Ann Thorac Surg. 2015;100:568-74.

31. Mohananey D, Sethi J, Vilalanca PA, et al. Effect of antiplatelet therapy on mortality and acute lung injury in critically ill patients. Ann Card Anaesth. 2016;19:626-37.

32. Martinez MN, Amidon GL. A mechanistic approach to understanding the factors affecting drug absorption: a review of fundamentals. J Clin Pharmacol. 2002;42:620-43.

33. Teng R, Carlson G, Hsia J. An open-label, randomized bioavailability study with alternative methods of administration of crushed ticagrelor tablets in healthy volunteers. Int J Clin Pharmacol Ther. 2015;53:182-9.

34. Dey P, Maiti S. Orodispersible tablets: a new trend in drug delivery. J Natl Sci Biol Med. 2010;1:2-5.

35. Bandari S, Mittapali RK, Gannu R, Rao YM. Orodispersible tablets: an overview. Asian J Pharmaceut. 2008;2:2-11.

36. Sillén H, Cook M, Davis P. Determination of ticagrelor and two metabolites in plasma samples by liquid chromatography and mass spectrometry. J Chromatogr B Analyt Technol Biomed Life Sci. 2010;878:2299-306.

37. Teng R, Butler K. Effect of CYP3A inhibitors, diltiazem and ketoconazole, on ticagrelor pharmacokinetics in healthy volunteers. J Drug Assess. 2013;2:30-9.

38. Food and Drugs Administration. Guidance for Industry: Bioavailability and bioequivalence studies for orally administered drug products—general considerations. March 2003. http://www. fda.gov/ohrms/dockets/ac/03/briefing/3995B1_07_GFI-BioAvailBioEquiv.pdf. Accessed 21 Feb 2016.

39. European Medicines Agency. Guideline on the investigation of bioequivalence. January 2010. http://www.ema.europa.eu/docs/ en_GB/document_library/Scientific_guideline/2010/01/WC5000 70039.pdf. Accessed $21 \mathrm{Feb} 2016$.

40. Japanese Pharmaceutical and Food Safety Bureau. Guideline for bioequivalence studies of generic products. February 2012. http:// www.nihs.go.jp/drug/be-guide(e)/Generic/GL-E_120229_BE.pdf. Accessed 21 Feb 2016. 
41. Teng R. Ticagrelor: pharmacokinetic, pharmacodynamic, and pharmacogenetic profile-an update. Clin Pharmacokinet. 2015;54:1125-38.

42. Crean B, Finnie C, Crosby A. Evaluation of crushed ticagrelor tablet doses: recovery following crushing and naso-gastric tube passage ex vivo. Drugs R D. 2013;13:153-7.

43. Alexopoulos D, Barampoutis N, Gkizas V, Vogiatzi C, Tsigkas $\mathrm{G}$, Koutsogiannis N, et al. Crushed versus integral tablets of ticagrelor in ST-segment elevation myocardial infarction patients: a randomized pharmacokinetic/pharmacodynamic study. Clin Pharmacokinet. 2016;55:359-67.

44. Asher E, Frydman S, Katz M, Regev E, Sabbag A, Mazin I, et al. Chewing versus swallowing ticagrelor to accelerate platelet inhibition in acute coronary syndrome-the CHEERS study. For the PLATIS (Platelets and Thrombosis in Sheba) Study Group. Thromb Haemost. 2017;117:727-33.
45. Asher E, Tal S, Mazin I, Katz M, Sabbag A, Regev E, et al. Chewing versus traditional swallowing of ticagrelor to accelerate platelet inhibition in ST elevation myocardial infarction-the CHEERS-STEMI study. JACC. 2017. doi:10.1016/S07351097(17)33410-1:21.

46. Venetsanos D, Sederholm Lawesson S, Swahn E, Alfredsson J. Chewed ticagrelor tablets provide faster platelet inhibition compared to integral tablets: the inhibition of platelet aggregation after administration of three different ticagrelor formulations (IPAAD-Tica) study, a randomised controlled trial. Thromb Res. 2017;149:88-94.

47. Parodi G, Xanthopoulou I, Bellandi B, Gkizas V, Valenti R, Karanikas S, et al. Ticagrelor crushed tablets administration in STEMI patients: the MOJITO study. J Am Coll Cardiol. 2015;65:511-2. 